\footnotetext{
${ }^{14}$ Y. Nambu and G. Jona-Lasino, Phys. Rev. 122, 345 (1961); S. Coleman and E. Weinberg, Phys. Rev. D 7 , 1888 (1973).

${ }^{15} \mathrm{~K}$. Symanzik (to be published) has recently suggested that one consider a $\lambda \varphi^{4}$ theory with a negative $\lambda$ to achieve UV stability at $\lambda=0$ 。 However, one can show, using the renormalization-g roup equations, that in such theory the ground-state energy is unbounded from below (S. Coleman, private communication).
}

${ }^{16} \mathrm{~W}$ 。A. Bardeen, H. Fritzsch, and M。Gell-Mann, CERN Report No. CERN-TH-1538, 1972 (to be published)。

${ }^{17} \mathrm{H}$. Georgi and S. L. Glashow, Phys. Rev. Lett. 28, 1494 (1972); S。Weinberg, Phys. Rev. D 5 , 1962 (1972). ${ }^{18}$ For a review of this program, see $S$ 。 Proceedings of the Sixteenth International Conference on High Energy Physics, National Accelerator Laboratory, Batavia, Illinois, 1972 (to be published).

\title{
Reliable Perturbative Results for Strong Interactions?*
}

\author{
H. David Politzer \\ Jefferson Physical Laboratories, Harvard University, Cambridge, Massachusetts 02138 \\ (Received 3 May 1973)
}

An explicit calculation shows perturbation theory to be arbitrarily good for the deep Euclidean Green's functions of any Yang-Mills theory and of many Yang-Mills theories with fermions. Under the hypothesis that spontaneous symmetry breakdown is of dynamical origin, these symmetric Green's functions are the asymptotic forms of the physically significant spontaneously broken solution, whose coupling could be strong.

Renormalization-group techniques hold great promise for studying short-distance and strongcoupling problems in field theory. ${ }^{1,2}$ Symanzik $^{2}$ has emphasized the role that perturbation theory might play in approximating the otherwise unknown functions that occur in these discussions. But specific models in four dimensions that had been investigated yielded (in this context) disappointing results. ${ }^{3}$ This note reports an intriguing contrary finding for any generalized Yang-Mills theory and theories including a wide class of fermion representations. For these one-coupling-constant theories (or generalizations involving product groups) the coefficient function in the Callan-Symanzik equations commonly called $\beta(g)$ is negative near $g=0$.

The constrast with quantum electrodynamics (QED) might be illuminating. Renormalization . of QED must be carried out at off-mass-shell points because of infrared divergences. For small $e^{2}$, we expect perturbation theory to be good in some neighborhood of the normalization point. But what about the inevitable logarithms of momenta that grow as we approach the mass shell or as some momenta go to infinity? In QED, the mass-shell divergences do not occur in observable predictions, when we take due account of the experimental situation. The renormalization-group technique ${ }^{4}$ provides a somewhat opaque analysis of this situation. Loosely speaking, ${ }^{5}$ the effective coupling of soft photons goes to zero, compensating for the fact that there are more and more of them. But the large$p^{2}$ divergence represents a real breakdown of perturbation theory. It is commonly said that for momenta such that $e^{2} \ln \left(p^{2} / m^{2}\right) \sim 1$, higher orders become comparable, and hence a calculation to any finite order is meaningless in this domain. The renormalization group technique shows that the effective coupling grows with momenta.

The behavior in the two momentum regimes is reversed in a Yang-Mills theory. The effective coupling goes to zero for large momenta, but as $p^{2}$ 's approach zero, higher-order corrections become comparable. Thus perturbation theory tells nothing about the mass-shell structure of the symmetric theory. Even for arbitrarily small $g^{2}$, there is no sense in which the interacting theory is a small perturbation on a free multiplet of massless vector mesons. The truly catastrophic infrared problem makes a symmetric particle interpretation impossible. Thus, though one can well approximate asymptotic Green's functions, to what particle states do they refer?

Consider theories defined by the Lagrangian

$$
\mathfrak{L}=-\frac{1}{4} F_{\mu \nu}^{a} F^{a \mu \nu}+i \bar{\psi}_{i} \gamma \cdot D_{i j} \psi_{j},
$$

where

$$
F_{\mu \nu}{ }^{a}=\partial_{\mu} A_{\nu}{ }^{a}-\partial_{\nu} A_{\mu}{ }^{a}+g f^{a b c} A_{\mu}{ }^{b} A_{\nu}{ }^{c},
$$


and

$$
D_{i j}{ }^{\mu}=\partial^{\mu} \delta_{i j}-i g A^{a \mu} T_{i j}{ }^{a},
$$

the $f^{a b c}$ are the group structure constants, and the $T^{a}$ are representation matrices corresponding to the fermion multiplet. (One may be interested in models with massless fermions because of their group structure or because they have the same asymptotic for $\mathrm{ms}^{6}$ as massive theories.) The normalizations of the conventionally defined irreducible vertices for $n$ mesons and $n^{\prime}$ fermions, $\Gamma^{n, n^{\prime}}$, must refer to some mass $M$. The renormalization-group equation reads

$$
\left(M \frac{\partial}{\partial M}+\beta(g) \frac{\partial}{\partial g}+n \gamma_{A}(g)+n^{\prime} \gamma_{\psi}(g)\right) \Gamma^{n, n^{0}}=0 .
$$

Putting it in this form makes use of the first available simplification, proper choice of gauge.
Equation (2) describes how finite renormalizations accompanied by a change in $g$ and a rescaling of the fields leave the $\Gamma^{n_{0} n^{\prime}}$ unchanged. Consider gauges defined by $\alpha$ in the zeroth-order propagator

$$
\Delta_{\mu \nu}\left(p^{2}\right)=\frac{-g_{\mu \nu}+p_{\mu} p_{\nu} / p^{2}}{p^{2}}+\alpha \frac{p_{\mu} p_{\nu}}{p^{4}} .
$$

The generalized Ward identities ${ }^{7}$ imply that there are no higher-order corrections to the longitudinal part. But if the fields are rescaled as in Eq. (2), $\alpha$ must be changed to leave $\Gamma^{2}$ invariant. Hence $\alpha$ should occur in Eq. (2) much as $g$ does, and one would have to study the $\Gamma^{n, n^{n}}$ for arbitrary $\alpha$ to determine the coefficient functions perturbatively. But for $\alpha=0$ initially, it remains zero under finite renormalizations; so it suffices to study the theory in a Landau gauge.

To first order, the meson inverse propagator is

$$
\Gamma_{\mu \nu}^{2 a b}(p,-p)=\delta^{a b}\left(-g_{\mu \nu} p^{2}+p_{\mu} p_{\nu}\right)\left[1+\left(\frac{13}{3} c_{1}-\frac{8}{3} c_{2}\right)(g / 4 \pi)^{2} \ln \left(-p^{2} / M^{2}\right)\right],
$$

where

$$
f_{a c d} f_{b c d}=2 c_{1} \delta_{a b}, \quad \operatorname{tr}\left(T^{a} T^{b}\right)=2 c_{2} \delta_{a b},
$$

and $c_{1}>_{0}$ and $c_{2} \geqslant 0$. [For SU(2), $c_{1}=1, c_{2}$ (isodoublet) $=\frac{1}{4}$, and $c_{2}$ (isotriplet) =1.] To first order (only), the fermion self-energy is proportional to the self-energy in massless QED, which vanishes in the Landau gauge. Similarly, the contribution to the fermion-vector three-point vertex correction proportional to the first-order QED correction needs no subtractions and contains no reference to $M$. Calculation of the remaining correction, involving the meson self-coupling, yields

$$
\Gamma_{\mu i j}{ }^{1_{0} 2 a}(0, p,-p)=g T_{i j}{ }^{a} \gamma_{\mu}\left[1-\frac{3}{2} c_{1}(g / 4 \pi)^{2} \ln \left(-p^{2} / M^{2}\right)\right] \text {. }
$$

Applying Eq. (2) to these functions at their normalization points yields

$$
\gamma_{\psi}(g)=0+O\left(g^{4}\right), \quad \gamma_{A}(g)=\left(\frac{13}{3} c_{1}-\frac{8}{3} c_{2}\right)(g / 4 \pi)^{2}+O\left(g^{4}\right), \quad \beta(g)=-\left(\frac{22}{3} c_{1}-\frac{8}{3} c_{2}\right) g(g / 4 \pi)^{2}+O\left(g^{5}\right) .
$$

It is also apparent, by inspecting the graphs, that to this order the coupling constants of product groups do not enter into each other's $\beta$ functions.

For the case where there are no fermions, the coefficient functions can be obtained by setting $c_{2}=0$. (Even though the fermion-vector vertex, which had been used implicitly to define $g$, is no longer present, it can be simulated by introducing two multiplets of spinor fields with the same group transformations but opposite statistics. The physical effects of internal fermions are canceled by the ghosts - spinor fields with Bose statistics.) Alternatively, one can study the corrections to the three-meson vertex. Define $F$ by

$$
\Gamma_{\lambda \mu \nu}^{3 a b c}(\phi,-p, 0)=f^{a b c}\left(p_{\lambda} g_{\mu \nu}+p_{\mu} g_{\nu \lambda}-2 p_{\nu} g_{\lambda \mu}\right) g F\left(p^{2} / M^{2}, g^{2}\right) \text {. }
$$

The normalization condition is $F\left(-1, g^{2}\right)=1$ (up to a phase convention.) To first order

$$
F=1+\frac{17}{6} c_{1}(g / 4 \pi)^{2} \ln \left(-p^{2} / M^{2}\right)
$$

which yields the same $\beta$ as described above.

The renormalization-group "improved" perturbation theory ${ }^{4_{0}}$ extends results valid near the normalization point by effectively moving that point. The improved vertex functions are con- structed from the straightforward perturbative ones, involving a momentum-scale-dependent effective coupling $g^{\prime}(g, t)$, where $t=\frac{1}{2} \ln \left(s / M^{2}\right)$ and $s$ sets the scale, e.g., $s=\sum\left(-p_{i}{ }^{2}\right) . g^{\prime}(g, t)$ is defined by

$$
\begin{aligned}
& \partial g^{\prime} / \partial t=\beta\left(g^{\prime}\right), \\
& g^{\prime}(g, 0)=g .
\end{aligned}
$$


For the approximate $\beta^{\prime}$ s derived above, $\beta=-b g^{3}$,

$$
g^{\prime 2} \approx g^{2} /\left(1+2 b g^{2} t\right)
$$

Thus for a pure meson theory or for theories including not too many fermions (in the sense that $\left.c_{2}<\frac{11}{4} c_{1}\right), g^{\prime}$ goes to zero for asymptotic momenta, i.e., $t \rightarrow \infty$. The $\Gamma^{n, n^{\prime}}$ show a well-defined slow approach to quasifree field values.

It is worth remembering that successive orders of perturbation theory give the behavior of $\beta$ for infinitesimal $g$ and, strictly speaking, say nothing about finite $g$. Making a polynomial fit to a perturbative result for $\beta$ is pure conjecture.

Hypothesizing that $\beta$ stays negative (at least into the domain of strong coupling constant) relates all theories defined by Eq. (1) [with $g$ less than the first zero of $\beta(g)$ ] to the model with $g$ arbitrarily small by a change in mass scale. They all share the same asymptotic Green's functions, differing only by how large is asymptotic.

To utilize this result, we make the following hypothesis: The gauge symmetry breaks down spontaneously as a result of the dynamics. Consequently, the fields obtain (in general massive) particle interpretation-the Higgs phenomenon. As yet, nothing is known about the particle spectrum, the low-energy dynamics, or particles describable only by composite fields. But the Callan-Symanzik analysis says that the asymptotic Green's functions for the "dressed" fundamental fermion and vector fields are the symmetric functions discussed above. ${ }^{8}$

[An alternative is to introduce fundamental scalar fields, in terms of which the group transformation properties of the vacuum can be stud ied. ${ }^{9}$ But these theories are not in general ultraviolet stable in terms of the additional coupling constants that must be introduced. Particular models which are ultraviolet stable as well as spontaneously asymmetric have been found. ${ }^{10}$ But gauge theories of fermions (only) have aesthetic attractions, including the possibility of a dynamical determination of the dimensionless coupling constant. ${ }^{9}$ ]

Hypotheses of this type go back to the work of Nambu and collaborators. ${ }^{11}$. In the renormalizable massless theories including scalars that have been studied, ${ }^{9}$ infrared instability is a necessary condition for spontaneous symmetry breakdown. ${ }^{12}$ The model of Nambu and Jona-Lasinio can be treated by the methods of Coleman and Wein- berg. ${ }^{9}$ The model is defined by

$$
\mathcal{L}=i \bar{\psi} \gamma \cdot \partial \psi+g_{0}\left[(\bar{\psi} \psi)^{2}-\left(\bar{\psi} \gamma_{5} \psi\right)^{2}\right]
$$

and the stipulation that the momentum integrals are cut off at some Euclidean mass squared $\Lambda^{2}$. Define a scalar

$$
\varphi(x)=g_{0} \Psi(x) \psi(x)
$$

and an analogous pseudoscalar, which one can do because of the cutoff. A study of the Green's functions in the one-loop approximation yields all the original results. But the existence of the vacuum-degenerate solution requires the dimensionless parameter characterizing the theory to satisfy $g_{0} \Lambda^{2}>2 \pi^{2}$. But this is the condition that the one-loop correction to fermion-fermion scattering be at least as important as the tree approximation.

The situation is similar in the renormalizable models. $\lambda \varphi^{4}$ is stable for small $\lambda$ because the one-loop corrections are small. But in massless scalar QED, photon-loop corrections of order $e^{4}$ can dominate over the lowest order $\varphi$ $\varphi$ scattering (order $\lambda$ ) for both $\lambda$ and $e$ arbitrarily small. The requirement is just that $\lambda<e^{4}$. In this light, the problem with the Nambu-JonaLasinio model is not its nonrenormalizability but that in the domain of large $g_{0} \Lambda^{2}$, where spontaneous breakdown is alleged to occur, higher loop corrections are likely to dominate. (In the framework of the original solution, more complex infinite chains and self-energy graphs dominate over the ones studied.) In theories defined by Eq. (1), composite scalar densities can also be defined and studied in perturbation theory. But the condition that the one-loop approximation imply vacuum degeneracy requires that the expansion parameter be large, rendering the application of perturbation theory suspect.

The author thanks Sidney Coleman and Erick Weinberg, who have offered insights and advice freely, and the latter especially for his help in the computations.

\footnotetext{
*Work supported in part by the U. S. Air Force Office of Scientific Research under Contract No. F44620-70C-0030.

${ }^{1}$ Of central importance is the work reviewed in $\mathrm{K}$. Wilson and J. Kogut, "The Renormalization Group and the $\epsilon$ Expansion" (to be published); K. Johnson and M. Baker, "Some Speculations on High Energy Quantum Electrodynamics" (to be published); S. Adler, Phys. Rev. D $\underline{5}, 3021$ (1972).

${ }^{2}$ K. Symanzik, DESY Report No. DESY 72/73, 1972
} 
(to be published), and references therein.

${ }^{3}$ A. Zee, "Study of the Renormalization Group for Small Coupling Constants" (to be published).

${ }^{4}$ N. N. Bogoliubov and D. V. Shirkov, Introduction to the Theory of Quantized Fields (Interscience, New York, 1959).

${ }^{5}$ Definitions of the relevant quantities will be given, but for the general theory and derivations see Refs. 1, 2, and 4; S. Coleman, in the Proceedings of the 1971 International Summer School "Ettore Majorana" (Academic, New York, to be published); S. Coleman and E. Weinberg, Phys. Rev. D 7, 1888 (1973), whose conventions we follow.

${ }^{6}$ Asymptotic refers to a particular set of Euclidean momenta as they are collectively scaled upward.

${ }^{7}$ E. g., B. W. Lee and J. Zinn-Justin, Phys. Rev. D 5, 3121 (1972); G. 't Hooft, Nucl. Phys. B33, 173
(1971), which also include details of Feynman rules, regularization, etc.

${ }^{8}$ Configurations where the symmetric theory has infrared singularities not present in the massive case are discussed in detail by Symanzik (Ref. 2) .

${ }^{9}$ Coleman and Weinberg, Ref. 5 .

${ }^{10}$ D. Gross and F. Wilczek, preceding Letter [Phys. Rev. Lett. 30 , 1343 (1973)].

${ }^{11} \mathrm{Y}$. Nambu and G. Jona-Lasinio, Phys. Rev. $\underline{122}, 345$ (1961).

${ }^{12} \lambda \varphi^{4}$ theory with $\lambda<0$ is ultraviolet stable (Ref. 2) and hence infrared unstable but cannot be physically interpreted in perturbation theory. Using the computations of Ref. 9, for $\lambda<0$ "improved" perturbation theory is arbitrarily good for large field strengths. In particular, the potential whose minimum determines the vacuum decreases without bound for large field.

\section{ERRATA}

PRECESSION OF POSITIVE MUONS IN NICKEL AND IRON. M. L. G. Foy, Neil Heiman, W. J. Kossler, and C. E. Stronach [Phys. Rev. Lett. 30, 1064 (1973)].

In the abstract, the last sentence which reads "... the internal fields are: $\simeq 150 \mathrm{G}$ for $\mathrm{Ni}$ and ..." should read "... the internal fields are: $\simeq 1500 \mathrm{G}$ for $\mathrm{Ni}$ and $\cdots . "$

HADRON PRODUCTION BY ELECTRON-POSITRON ANNIHILATION AT 4-GeV CENTER- OFMASS ENERGY. A. Litke, G. Hanson, A. Hofmann, J. Koch, L. Law, M. E. Law, J. Leong, R. Little, R. Madaras, H. Newman, J. M. Paterson, R. Pordes, K. Strauch, G. Tarnopolsky, and Richard Wilson [Phys. Rev. Lett. 30, 1189 (1973)].

In press, a sentence was truncated on page 1190, second column, first paragraph. It should read "... 31 two-prong, 29 three-prong, 20 four-prong, and 8 five-prong events." 